\title{
Reflection properties of zeta related functions in terms of fractional derivatives
}

\author{
E. Ferreira* \\ Instituto de Física, Universidade Federal do Rio de Janeiro, \\ 21941-972, Rio de Janeiro, Brasil,
}

\author{
A. K. Kohara ${ }^{\dagger}$ \\ Centre de Physique Théorique, École Polytechnique, CNRS, \\ Université Paris-Saclay, F-91128, Palaiseau, France, \\ J. Sesma ${ }^{\ddagger}$ \\ Departamento de Física Teórica, Facultad de Ciencias, \\ 50009, Zaragoza, Spain.
}

June 28, 2020

\begin{abstract}
We prove that the Weyl fractional derivative is a useful instrument to express certain properties of the zeta related functions. Specifically, we show that a known reflection property of the Hurwitz zeta function $\zeta(n, a)$ of integer first argument can be extended to the more general case of $\zeta(s, a)$, with complex $s$, by replacement of the ordinary derivative of integer order by Weyl fractional derivative of complex order. Besides, $\zeta(s, a)$ with $\Re(s)>2$ is essentially the Weyl $(s-2)$-derivative of $\zeta(2, a)$. These properties of the Hurwitz zeta function can be immediately transferred to a family of polygamma functions of complex order defined in a natural way. Finally, we discuss the generalization of a recently unveiled reflection property of the Lerch's transcendent.
\end{abstract}

AMS Subject Classification:: Primary 26A33; Secondary 11M35, 33B15

Key Words and Phrases: Weyl fractional derivative, Hurwitz zeta function, polygamma function, Lerch's transcendent

\footnotetext{
*Email: erasmo@if.ufrj.br

${ }^{\dagger}$ Email: kendi@if.ufrj.br

${ }^{\ddagger}$ Corresponding author. Email: javier@unizar.es
} 


\section{Introduction}

Fractional calculus has become, in the last decades, a very fertile field of research. The historical survey by Machado and Kiryakova [17] allows to realize the interest arisen by both the foundations and the applications of fractional integrals and derivatives. As shown in [17], besides a considerable number of specialized monographs and proceedings dealing with different applications, treatises about general aspects of fractional calculus have been published. As an introduction for a non-familiar reader, we recommend a paper by Hilfer [12]. For a more sound and encyclopedic knowledge of the field, the book by Samko, Kilbas and Marichev [19] should be a must. Tables of the different definitions of fractional derivatives and of their application to a collection of functions can be found in [20].

A large variety of special functions can be expressed as fractional differintegrals of ordinary functions. Fractional calculus has proved to be a very useful tool also to unveil certain properties of those functions $[13,14,15,16]$. Here we present a new example of such kind of applications of fractional differentiation.

In a recent paper [8], a "symmetry" property of the Lerch's transcendent, namely

$$
\begin{aligned}
&(-1)^{n-1} \Phi(z, n, a)-z^{-1} \Phi\left(z^{-1}, n, 1-a\right)= \\
& \frac{\pi}{(n-1) !} \frac{\partial^{n-1}}{\partial a^{n-1}}\left(z^{-a}(\cot (\pi a)+\operatorname{sgn}(\arg (z)) i)\right),
\end{aligned}
$$

where sgn represents the "sign" function, was proved for complex values of $z$ and $a$ and positive integer values of the second argument, $n=1,2, \ldots$ The possible extension of this property to more general values of that argument was not considered, as it required to introduce fractional derivatives, a subject that lay out of the scope of that paper. Here, we deal with this issue. We try to extend the mentioned property, with an adequate formulation, to complex values $s$ of the second argument, with the only restriction that $\Re(s)>1$.

To start with, we consider a particular case of Lerch's transcendent, namely the Hurwitz zeta function $\zeta(s, a)$, which results by taking $z=1$ in $\Phi(z, s, a)[18$, Eq. 25.14.2],

$$
\zeta(s, a)=\Phi(1, s, a), \quad \Re(s)>1, \quad a \neq 0,-1,-2, \ldots .
$$

It is well known $[4$, Sec. 4] that the Hurwitz zeta function, for positive integer values of its first argument, possesses the reflection property

$$
(-1)^{n-1} \zeta(n, a)-\zeta(n, 1-a)=\frac{\pi}{(n-1) !} \frac{d^{n-1}}{d a^{n-1}} \cot (\pi a), \quad n=2,3, \ldots,
$$

counterpart of (1). Such reflection property results from the relation of the Hurwitz zeta function with a polygamma function [18, Eq. 25.11.12],

$$
\zeta(n, a)=\frac{(-1)^{n}}{(n-1) !} \psi^{(n-1)}(a),
$$


and the fact that the polygamma functions obey the reflection property $[18$, Eq. 5.15.6]

$$
(-1)^{m-1} \psi^{(m)}(a)+\psi^{(m)}(1-a)=(-1)^{m} \pi \frac{d^{m}}{d a^{m}} \cot (\pi a), \quad m=0,1,2, \ldots,
$$

a relation which follows trivially from the reflection property of the Gamma function [18, Eq. 5.5.3]

$$
\Gamma(a) \Gamma(1-a)=\pi / \sin (\pi a) .
$$

We show, in Section 2, that a relation extending (3) to complex values $s$ of the first argument of the Hurwitz zeta function can be formulated in terms of fractional derivatives (of complex order), whenever $\Re(s)>1$. Section 3 shows that it is possible to define polygamma functions of complex order, so as to obtain a generalization of (5), with $m$ replaced by a complex number $s$. In Section 4 we discuss the possible extension of our published result (1) to complex values of the second argument of the Lerch's transcendent. Finally, Section 5 contains some comments about the results presented in this paper.

Among the different possibilities that have been proposed, we find convenient for our purposes to adopt the Weyl definition for fractional derivative, as presented in [3, Secs. 1.2 and 1.3]. Accordingly, the fractional derivative of (complex) order $\alpha$ of a function $f(x)$ is given by

$$
{ }_{x} D_{\infty}^{\alpha} f(x) \equiv \frac{(-1)^{m}}{\Gamma(m-\alpha)} \frac{d^{m}}{d x^{m}} \int_{x}^{\infty}(t-x)^{m-\alpha-1} f(t) d t,
$$

where $m$ is the integer such that $m-1 \leq \Re(\alpha)<m$. (Notice that the Weyl definition for fractional derivative given in [7] is different from that in [3] by a factor $(-1)^{m}$.) The path of integration is one of the rays $t=x r$ with $r>1$, or $t=x+r$ with $r>0$. Then, for the particular cases of $f(x)$ to be considered in this paper, one has

$$
\begin{aligned}
{ }_{x} D_{\infty}^{\alpha}(x+a)^{-\lambda}= & \frac{\Gamma(\lambda+\alpha)}{\Gamma(\lambda)}(x+a)^{-\lambda-\alpha}, \\
& 0<m-\Re(\alpha)<\Re(\lambda), \quad|\arg (x / a)|<\pi, \\
{ }_{x} D_{\infty}^{\alpha} e^{p x}= & (-p)^{\alpha} e^{p x}, \quad \Re(p x)<0 .
\end{aligned}
$$

A few words about notation are in order. In what follows, $s$ and $n$ represent, respectively, a complex and an integer number such that

$$
n-1 \leq \Re(s)<n .
$$

To avoid ambiguous expressions of the type $(-1)^{b}$, with non-integer $b$, we shall use a symbol $\sigma$, depending on two (complex) parameters $a$ and $b$, whose values are given by

$$
\sigma(a, b) \equiv \begin{cases}e^{i \pi b} & \text { if } \quad \Im(a)>0, \\ e^{-i \pi b} & \text { if } \Im(a)<0 .\end{cases}
$$


Obviously,

$$
\begin{gathered}
\sigma(-a, b)=\sigma(a,-b)=(\sigma(a, b))^{-1} \\
\sigma(a+r, b)=\sigma(a, b) \quad \text { for real } r \\
\sigma(a, b \pm 1)=-\sigma(a, b), \quad \sigma(a, b \pm 2)=\sigma(a, b) \\
(\sigma(a, b))^{c}=\sigma(a, b c)
\end{gathered}
$$

\section{Reflection property of the Hurwitz zeta func- tion}

The Hurwitz zeta function $\zeta(s, a)$ is defined by the series expansion [18, Eq. 25.11.1]

$$
\zeta(s, a)=\sum_{m=0}^{\infty} \frac{1}{(a+m)^{s}}, \quad \Re(s)>1, \quad a \neq 0,-1,-2, \ldots .
$$

It admits different integral representations [18, §25.11] which allow to extend its definition to other ranges of values of its arguments. Here we will make reference to one of those integral representations, namely [18, Eq. 25.11.25]

$$
\zeta(s, a)=\frac{1}{\Gamma(s)} \int_{0}^{\infty} \frac{x^{s-1} e^{-a x}}{1-e^{-x}} d x, \quad \Re(s)>1, \quad \Re(a)>0 .
$$

The reflection property (3) can be extended according to the following

Proposition 1. The Hurwitz zeta function $\zeta(s, a)$ of complex s such that $\Re(s)>1$ and complex a such that $\Im(a) \neq 0$ satisfies the reflection property

$$
\sigma(a, s-1) \zeta(s, a)-\zeta(s, 1-a)=\frac{\pi}{\Gamma(s)} \sigma(a, s-1){ }_{a} D_{\infty}^{s-1}(\cot (\pi a)+\sigma(a, 1 / 2)),
$$

the symbol $\sigma$ being defined in (11).

Proof. Let us restrict, for the moment, the values of $a$ to the band $0<$ $\Re(a)<1$. We consider, in the first place, the case of $\Im(a)>0$. Designing by $\mathcal{C}^{+}$ the closed path in the complex $t$ plane formed by the real axis and an infinite radius semi-circumference (sc), in the upper half-plane, going counter-clock-wise from $+\infty$ to $-\infty$, we have

$$
\int_{\mathcal{C}^{+}} \frac{t^{s-1} e^{a t}}{e^{t}-1} d t=I_{1}+I_{2}-I_{3}
$$

where

$$
I_{1}=\int_{0}^{+\infty} \frac{t^{s-1} e^{a t}}{e^{t}-1} d t, \quad I_{2}=\int_{s c} \frac{t^{s-1} e^{a t}}{e^{t}-1} d t, \quad I_{3}=\int_{0}^{\infty e^{i \pi}} \frac{t^{s-1} e^{a t}}{e^{t}-1} d t .
$$


The integrand in the left-hand side of (15) presents single poles at the points

$$
t_{k} \equiv 2 k \pi i, \quad k=0, \pm 1, \pm 2, \ldots,
$$

the respective residues being

$$
t_{k}^{s-1} e^{a t_{k}}=(2 k \pi i)^{s-1} e^{2 k \pi i a} .
$$

Then, obviously,

$$
\int_{\mathcal{C}^{+}} \frac{t^{s-1} e^{a t}}{e^{t}-1} d t=2 \pi i \sum_{k=1}^{\infty} t_{k}^{s-1} e^{a t_{k}} .
$$

On the other hand, bearing in mind the representation (13) of the Hurwitz zeta function, one has

$$
\begin{aligned}
& I_{1}=\int_{0}^{+\infty} \frac{t^{s-1} e^{-(1-a) t}}{1-e^{-t}} d t=\Gamma(s) \zeta(s, 1-a), \\
& I_{2}=0, \quad \text { assumed that } \quad 0<\Re(a)<1, \\
& I_{3}=\int_{0}^{+\infty} \frac{\left(r e^{i \pi}\right)^{s-1} e^{-a r}}{e^{-r}-1}(-d r)=e^{i \pi(s-1)} \Gamma(s) \zeta(s, a) .
\end{aligned}
$$

Substituting the terms in (15) by their values as given in Eqs. (19) to (22), one obtains

$$
e^{i \pi(s-1)} \zeta(s, a)-\zeta(s, 1-a)=-\frac{\pi i}{\Gamma(s)} 2 \sum_{k=1}^{\infty} t_{k}^{s-1} e^{a t_{k}} .
$$

In view of the fractional derivation rule (9), the last equation can be written in the form

$$
e^{i \pi(s-1)} \zeta(s, a)-\zeta(s, 1-a)=-\frac{\pi i}{\Gamma(s)} e^{i \pi(s-1)}{ }_{a} D_{\infty}^{s-1}\left(2 \sum_{k=1}^{\infty} e^{a t_{k}}\right) .
$$

By substituting the values of the $t_{k}$, given in (17), one has, in the present case of $\Im(a)>0$,

$$
e^{i \pi(s-1)} \zeta(s, a)-\zeta(s, 1-a)=-\frac{\pi i}{\Gamma(s)} e^{i \pi(s-1)}{ }_{a} D_{\infty}^{s-1}\left(2 \sum_{k=1}^{\infty} e^{2 k \pi i a}\right) .
$$

Let us consider now the case of $\Im(a)<0$. By taking the complex conjugate of (25) (denoting now $s$ and $a$ the complex conjugate of their values in (25)) we obtain

$$
e^{-i \pi(s-1)} \zeta(s, a)-\zeta(s, 1-a)=\frac{\pi i}{\Gamma(s)} e^{-i \pi(s-1)}{ }_{a} D_{\infty}^{s-1}\left(2 \sum_{k=1}^{\infty} e^{-2 k \pi i a}\right) .
$$

Alternatively, (26) can be proved by choosing a closed path $\mathcal{C}^{-}$symmetric of $\mathcal{C}^{+}$ with respect to the real axis and following a procedure analogous to that from 
Eqs. (15) to Eq. (25). Recalling that

$$
\cot (\pi a)= \begin{cases}-2 i \sum_{k=1}^{\infty} e^{2 k \pi i a}-i & \text { for } \quad \Im(a)>0, \\ 2 i \sum_{k=1}^{\infty} e^{-2 k \pi i a}+i & \text { for } \quad \Im(a)<0,\end{cases}
$$

both Eqs. (25) and (26) can be written in the form (14).

To end the proof, it lacks to show that the result (14), just proved for $0<\Re(a)<1$, holds for more general values of $a$. Using the property [18, Eq. 25.11.4]

$$
\zeta(s, a)=\zeta(s, a+m)+\sum_{j=0}^{m-1} \frac{1}{(j+a)^{s}},
$$

the result can be extended to values of $a$ in any band $m<\Re(a)<m+1$, $m= \pm 1, \pm 2, \ldots$. Continuity across the lines $\Re(a)=m, m=0, \pm 1, \pm 2, \ldots$, allows to complete the proof.

Remark 1. It is known that a constant has not a defined Weyl derivative. Nevertheless, the sum $\cot (\pi a)+\sigma(a, 1 / 2)$ appearing in the right-hand side of (14) admits a fractional derivative, in the Weyl sense, whenever $\Im(a) \neq 0$. The representation (27) allows to prove immediately the existence of such derivative. That representation can also be used for its numerical computation. The resulting series converges as a geometric one, the ratio of the absolute value of an advanced term to that of the preceding one being approximately $\exp (-2 \pi|\Im(a)|)$. Obviously, this series, although convergent, is not useful from the numerical point of view in the case of very small $|\Im(a)|$.

Remark 2. It is immediate to see that our relation (14) turns into the well known (3) in the case of $s$ being an integer, $s=n=2,3, \ldots$. (Remember that, for positive integer $m,{ }_{x} D_{\infty}^{m} f(x)=(-1)^{m} d^{m} f(x) / d x^{m}$.)

Remark 3. According to the fractional derivation rule (8), it becomes evident that

$$
\zeta(s, a)=\frac{1}{\Gamma(s)}{ }_{a} D_{\infty}^{s-2} \zeta(2, a), \quad \zeta(s, 1-a)=\frac{1}{\Gamma(s)} \sigma(a, s-2){ }_{a} D_{\infty}^{s-2} \zeta(2,1-a),
$$

for $\Re(s)>2$. Our result (14) shows that it would be erroneous to conclude that

$$
\sigma(a, s-1) \zeta(s, a)-\zeta(s, 1-a)=\frac{\pi}{\Gamma(s)} \sigma(a, s-1){ }_{a} D_{\infty}^{s-1} \cot (\pi a),
$$

as it appears by applying, in a naive way, ${ }_{a} D_{\infty}^{s-2}$ to the two sides of the relation

$$
-\zeta(2, a)-\zeta(2,1-a)=\pi \frac{d}{d a} \cot (\pi a),
$$

that is a particular case of (3). However, the correct result is obtained when ${ }_{a} D_{\infty}^{s-2}$ operates on both sides of

$$
-\zeta(2, a)-\zeta(2,1-a)=\pi \frac{d}{d a}(\cot (\pi a)+\sigma(a, 1 / 2))
$$


a true relation equivalent to (31) in most aspects, but not as object of Weyl fractional derivation.

\section{Polygamma functions of complex order}

The successive integer derivatives of the logarithm of the Gamma function are known as polygamma functions [1, Eq. 6.4.1],

$$
\psi^{(m)}(a)=\frac{d^{m+1}}{d a^{m+1}} \ln \Gamma(a), \quad(m=0,1,2, \ldots) .
$$

For $m>0$, they are closely related to particularizations of the Hurwitz zeta function with integer first argument [18, Eq. 25.11.12], as shown in (4), that can be written in the form

$$
\psi^{(m)}(a)=(-1)^{m+1} m ! \zeta(m+1, a), \quad(m=1,2,3, \ldots) .
$$

The problem of defining polygamma functions $\psi^{(s)}$ of order $s$ different from a non-negative integer has been considered by several authors $[2,5,9,10]$. Here we adopt a definition of $\psi^{(s)}$ with complex $s(\Re(s)>0)$ which is a natural extension of the relation (34).

Definition 1. The polygamma functions $\psi^{(s)}$ of complex order $s$ such that $\Re(s)>0$ and complex argument $a$ such that $\Im(a) \neq 0$ are defined by the relation

$$
\psi^{(s)}(a)=\sigma(a, s+1) \Gamma(s+1) \zeta(s+1, a), \quad \Re(s)>0 .
$$

As it follows from (14) and (35), the so defined polygamma functions obey, in the case of $\Im(a) \neq 0$, the reflection property

$$
\psi^{(s)}(a)-\sigma(a, s) \psi^{(s)}(1-a)=-\sigma(a, s) \pi_{a} D_{\infty}^{s}(\cot (\pi a)+\sigma(a, 1 / 2)),
$$

which, in the case of integer $s=m$, becomes

$$
\psi^{(m)}(a)-(-1)^{m} \psi^{(m)}(1-a)=-\pi \frac{d^{m}}{d a^{m}} \cot (\pi a),
$$

in accordance with (5).

Remark 4. Notice that the definition (35) is compatible with this other one

$$
\psi^{(s)}(a)=\sigma(a, s-1){ }_{a} D_{\infty}^{s-1} \psi^{(1)}(a), \quad \Re(s)>1,
$$

that is a natural extension of (33). To check the compatibility, let us recall the series expansion of the trigamma function [18, Eq. 5.15.1]

$$
\psi^{(1)}(a)=\frac{d^{2}}{d a^{2}} \ln \Gamma(a)=\sum_{k=0}^{\infty} \frac{1}{(k+a)^{2}} .
$$


Then, from (38) and (39), applying the derivation rule (8), we obtain

$$
\begin{aligned}
\psi^{(s)}(a) & =\sigma(a, s-1) \Gamma(s+1) \sum_{k=0}^{\infty} \frac{1}{(k+a)^{s+1}} \\
& =\sigma(a, s-1) \Gamma(s+1) \zeta(s+1, a),
\end{aligned}
$$

to be compared with (35).

\section{Reflection property of the Lerch's transcen- dent}

We recall that the Lerch's transcendent $\Phi(z, s, a)$, also known as Hurwitz-Lerch zeta function, is defined by its series representation [6, Sec. 1.11, Eq. (1)] [18, Eq. 25.14.1]

$$
\Phi(z, s, a)=\sum_{m=0}^{\infty} \frac{z^{m}}{(a+m)^{s}}
$$

provided

$$
a \neq 0,-1,-2, \ldots ; \quad|z|<1 ; \quad|z|=1, \quad \Re(s)>1 .
$$

For other values of its arguments, $\Phi(z, s, a)$ is defined by analytic continuation. This is achieved by means of integral representations, the most common of them being [18, Eq. 25.14.5]

$$
\Phi(z, s, a)=\frac{1}{\Gamma(s)} \int_{0}^{\infty} \frac{t^{s-1} e^{-a t}}{1-z e^{-t}} d t
$$

whenever

$$
\Re(s)>0, \quad \Re(a)>0, \quad z \in \mathbb{C} \backslash[1, \infty) .
$$

The equivalence of the representations (41) and (43) in their common region of validity can be seen in, for instance, [11, Lemmas 2.1 and 2.2].

In analogy with the case of the Hurwitz zeta function, discussed in Sec. 2, we state the following

Proposition 2. Let $\mathbf{D}$ denote the open unit disc in the complex plane, cut along the negative real semiaxis, that is,

$$
z \in \mathbf{D} \quad \Rightarrow \quad z \in \mathbb{C}, \quad 0<|z|<1, \quad-\pi<\arg (z)<\pi .
$$

For $z \in \mathbf{D} \backslash(0,1)$, complex $s$ such that $\Re(s)>1$, and complex a such that $\Im(a) \neq 0$, the Lerch's transcendent presents the "symmetry" property

$$
\begin{aligned}
& \sigma(a, s-1) \Phi(z, s, a)-z^{-1} \Phi\left(z^{-1}, s, 1-a\right)= \\
& \begin{cases}\frac{\pi i}{\Gamma(s)}\left(-2 \sum_{k=0}^{\infty} t_{k}^{s-1} e^{a t_{k}}+\operatorname{sgn}(\arg (z)) t_{0}^{s-1} e^{a t_{0}}\right), & \text { for } \Im(a)>0, \\
\frac{\pi i}{\Gamma(s)}\left(2 \sum_{k=0}^{\infty}{ }^{\prime} t_{-k}^{s-1} e^{a t_{-k}}+\operatorname{sgn}(\arg (z)) t_{0}^{s-1} e^{a t_{0}}\right), & \text { for } \Im(a)<0,\end{cases}
\end{aligned}
$$


with the notation (5) for the symbol $\sigma$ and being now

$$
t_{k} \equiv-\ln z+2 k \pi i, \quad k=0, \pm 1, \pm 2, \ldots .
$$

The "prime" at the summation sign indicates that the term in the sum corresponding to $k=0$ must be halved.

Proof. The demonstration of this property runs along the same steps as those of Proposition 2.1. To start with, it is assumed that $0<\Re(a)<1$ and $\Im(a)>0$. Being the closed path $\mathcal{C}^{+}$the same as before, one has

$$
\int_{\mathcal{C}^{+}} \frac{t^{s-1} e^{a t}}{z e^{t}-1} d t=I_{1}+I_{2}-I_{3}
$$

where now

$$
I_{1}=\int_{0}^{+\infty} \frac{t^{s-1} e^{a t}}{z e^{t}-1} d t, \quad I_{2}=\int_{s c} \frac{t^{s-1} e^{a t}}{z e^{t}-1} d t, \quad I_{3}=\int_{0}^{\infty e^{i \pi}} \frac{t^{s-1} e^{a t}}{z e^{t}-1} d t
$$

The integrand in the left-hand side of (48) presents single poles at the points $t_{k}$ given in (47), the respective residues being now

$$
t_{k}^{s-1} e^{a t_{k}}=(-\ln z+2 k \pi i)^{s-1} z^{-a} e^{2 k \pi i a} .
$$

In the present case,

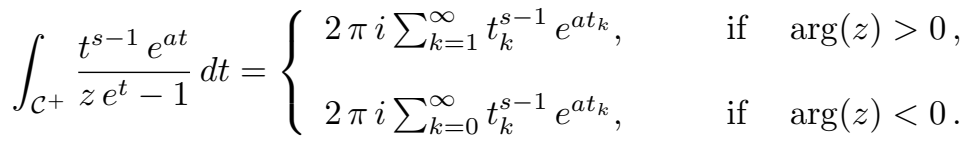

In view of the representation (43) of the Lerch's transcendent, one may write

$$
\begin{aligned}
& I_{1}=z^{-1} \int_{0}^{+\infty} \frac{t^{s-1} e^{-(1-a) t}}{1-z^{-1} e^{-t}} d t=z^{-1} \Gamma(s) \Phi\left(z^{-1}, s, 1-a\right), \\
& I_{2}=0, \quad \text { since } 0<\Re(a)<1, \\
& I_{3}=\int_{0}^{+\infty} \frac{\left(r e^{i \pi}\right)^{s-1} e^{-a r}}{z e^{-r}-1}(-d r)=e^{i \pi(s-1)} \Gamma(s) \Phi(z, s, a) .
\end{aligned}
$$

Then, Eq. (48) becomes

$$
\begin{aligned}
& e^{i \pi(s-1)} \Phi(z, s, a)-z^{-1} \Phi\left(z^{-1}, s, 1-a\right)= \\
& -\frac{\pi i}{\Gamma(s)}\left(2 \sum_{k=0}^{\infty} t_{k}^{s-1} e^{a t_{k}}-\operatorname{sgn}(\arg (z)) t_{0}^{s-1} e^{a t_{0}}\right) .
\end{aligned}
$$

In the case of $0<\Re(a)<1$, as before, but $\Im(a)<0$, one considers the closed path $\mathcal{C}^{-}$, symmetric of $\mathcal{C}^{+}$with respect to the real axis. Following a procedure analogous to that from Eqs. (48) to Eq. (55) one arrives in this case to

$$
\begin{aligned}
& e^{-i \pi(s-1)} \Phi(z, s, a)-z^{-1} \Phi\left(z^{-1}, s, 1-a\right)= \\
& \frac{\pi i}{\Gamma(s)}\left(2 \sum_{k=0}^{\infty} t_{-k}^{s-1} e^{a t_{-k}}+\operatorname{sgn}(\arg (z)) t_{0}^{s-1} e^{a t_{0}}\right) .
\end{aligned}
$$


Both Eqs. (55) and (56) can be unified to give (46)

It lacks to show that the result (46), just proved for $a$ in the band $0<\Re(a)<$ 1 , holds also for $a$ out of this band. By using the property [18, Eq. 25.14.4]

$$
\Phi(z, s, a)=z^{m} \Phi(z, s, a+m)+\sum_{n=0}^{m-1} \frac{z^{n}}{(a+n)^{s}},
$$

the result can be extended to values of $a$ in any band $m<\Re(a)<m+1$, $m= \pm 1, \pm 2, \ldots$. The proof is completed by invoking continuity across the lines $\Re(a)=m, m=0, \pm 1, \pm 2, \ldots$

Remark 5. In view of the fractional derivation rule (9), one is tempted to replace in the right-hand sides of (55) and (56)

$$
t_{k}^{s-1} e^{a t_{k}} \quad \text { by } \quad \sigma(a, s-1){ }_{a} D_{\infty}^{s-1} e^{a t_{k}}, \quad k=0, \pm 1, \pm 2, \ldots
$$

in order to obtain for the reflection property

$$
\begin{aligned}
& \sigma(a, s-1) \Phi(z, s, a)-z^{-1} \Phi\left(z^{-1}, s, 1-a\right)= \\
& \quad \sigma(a, s-1) \frac{\pi}{\Gamma(s)}{ }_{a} D_{\infty}^{s-1}\left(z^{-a}(\cot (\pi a)+\operatorname{sgn}(\arg (z)) i)\right),
\end{aligned}
$$

in consonance with (1). However, the replacement (58) is not always justified. It requires the fulfilment of $\Re\left(a t_{0}\right)<0$, a severe restriction on the values of $a$. Nevertheless, the relation (59) can be accepted as purely formal, understanding that, in any application of it, $\cot (\pi a)$ should be replaced by the corresponding right-hand side of (27) and the derivation rule (9) should be used, irrespective of the sign of the real part of the exponent.

Remark 6. It is immediate to verify that Eq. (46) remains invariant under the simultaneous replacements of $z$ by $z^{-1}$ and $a$ by $(1-a)$. Let us define, to avoid confusions,

$$
w \equiv z^{-1}, \quad b \equiv 1-a, \quad \hat{t}_{k} \equiv-\ln w+2 k \pi i,
$$

with $z$ as assumed in Proposition 4.1. Obviously, $\arg (w)=-\arg (z)$. Then, (46) can be written in the form

$$
\begin{aligned}
& \sigma(b, s-1) \Phi(w, s, b)-w^{-1} \Phi\left(w^{-1}, s, 1-b\right)= \\
& \begin{cases}\frac{\pi i}{\Gamma(s)}\left(-2 \sum_{k=0}^{\infty} \hat{t}_{k}^{s-1} e^{b \hat{t}_{k}}+\operatorname{sgn}(\arg (w)) \hat{t}_{0}^{s-1} e^{b \hat{t}_{0}}\right), & \text { for } \Im(b)>0, \\
\frac{\pi i}{\Gamma(s)}\left(2 \sum_{k=0}^{\infty} \hat{t}_{-k}^{s-1} e^{b \hat{t}_{-k}}+\operatorname{sgn}(\arg (w)) \hat{t}_{0}^{s-1} e^{b \hat{t}_{0}}\right), & \text { for } \Im(b)<0,\end{cases}
\end{aligned}
$$

where

$$
|w|>1, \quad w \notin(-\infty, 1) \cup(1, \infty),
$$

This means that, although it has been deduced on the assumption that $|z|<1$, the property (46) applies, as it is, to the case of $|z|>1$, provided $z \notin(-\infty, 1) \cup$ 
$(1, \infty)$, and is also valid, by continuation, for $|z|=1$. The interesting particular value $z=1$ has already been considered in Sec. 2 .

Remark 7. The relation (61) offers the possibility of writing an expansion of $\Phi(w, s, b)$, as a series of powers of $w^{-1}$, convergent for $|w|>1$ and $\Re(s)>1$. It follows immediately by rearranging terms and using the representation (41) for $\Phi\left(w^{-1}, s, 1-b\right)$ in (61). The expansion reads

$$
\begin{aligned}
\Phi(w, s, b)= & -\sum_{m=1}^{\infty} \frac{w^{-m}}{(b-m)^{s}}+\frac{\pi i}{\Gamma(s)} \operatorname{sgn}(\arg (w))\left(-\hat{t}_{0}\right)^{s-1} e^{b \hat{t}_{0}} \\
& + \begin{cases}-\frac{\pi i}{\Gamma(s)} 2 \sum_{k=0}^{\infty}{ }^{\prime}\left(-\hat{t}_{k}\right)^{s-1} e^{b \hat{t}_{k}}, & \text { for } \Im(b)>0, \\
\frac{\pi i}{\Gamma(s)} 2 \sum_{k=0}^{\infty}{ }^{\prime}\left(-\hat{t}_{-k}\right)^{s-1} e^{b \hat{t}_{-k}}, & \text { for } \Im(b)<0,\end{cases}
\end{aligned}
$$

the $\hat{t}_{k}$ being given in $(60)$.

\section{$5 \quad$ Final comments}

It was well known that the zeta related functions, namely $\zeta(n, a), \psi^{(n)}(a)$ and $\Phi(z, n, a)$ possess a certain reflection property in the case of integer values of the parameter $n$. In the preceding sections we have proved that such property can be extended to the case of complex values of this parameter. The resulting relations for $\zeta(s, a), \psi^{(s)}(a)$ and $\Phi(z, s, a)$, with complex $s(\Re(s)>1)$, adopt very simple forms in terms of Weyl fractional derivatives. It appears that such derivative is a very useful instrument to deal with functions of this type. In fact, from (8) and (9) and the definitions (41) and (43) of the Lerch's transcendent, it follows immediately that

$$
\Gamma(s) \Phi(z, s, a)={ }_{a} D_{\infty}^{s-1} \Phi(z, 1, a), \quad \Re(s)>1,
$$

a relation which can be extended to the half-plane $\Re(s)>0$ if the usual conventions [3, Sec. 1.2]

$$
\begin{array}{rlr}
{ }_{x} D_{\infty}^{\alpha} f(x) & =\frac{1}{\Gamma(-\alpha)} \int_{x}^{\infty}(t-x)^{-\alpha-1} f(t) d t, \quad \Re(\alpha)<0, \\
{ }_{x} D_{\infty}^{i \theta} f(x) & =\frac{-1}{\Gamma(1-i \theta)} \frac{d}{d x} \int_{x}^{\infty}(t-x)^{-i \theta} f(t) d t, \quad \text { real } \theta,
\end{array}
$$

are assumed in the cases of derivatives of order having negative real part or being purely imaginary.

In the study of the properties of the mentioned functions, we have been compelled to leave out the interesting case of real values of the argument $a$. We have not found a natural way of including that case, and the problem remains open. 


\section{Acknowledgements}

This work has been supported by Conselho Nacional de Desenvolvimento Científico e Tecnológico (CNPq, Brazil), by Coordenação de Aperfeiçoamento do Pessoal de Nível Superior (CAPES, Brazil) and by Departamento de Ciencia, Tecnología y Universidad del Gobierno de Aragón (Project E24/1) and Ministerio de Ciencia e Innovación (Project MTM2009-11154).

\section{References}

[1] M. Abramowitz, I.A. Stegun, Handbook of Mathematical Functions. Dover, New York (1965).

[2] V.S. Adamchik, Polygama functions of negative order. J. Comput. Appl. Math. 100 (1998), 191-199.

[3] P.L. Butzer, U. Westphal, An introduction to fractional calculus. In: $A p$ plications of Fractional Calculus in Physics, World Scientific, Singapore (2000), 1-85.

[4] D. Cvijović, J. Klinowski, Values of the Legendre chi and Hurwitz zeta functions at rational arguments. Math. Comp. 68 (1999), 1623-1630.

[5] O. Espinosa, V.H. Moll, A generalized polygamma function. Integral Transforms Spec. Funct. 15, No 2 (2004), 101-115.

[6] A. Erdélyi, W. Magnus, F. Oberhettinger, F.G. Tricomi. Higher Transcendental Functions. McGraw-Hill, New York (1953), Vol. 1.

[7] A. Erdélyi, W. Magnus, F. Oberhettinger, F.G. Tricomi. Tables of Integral Transforms. McGraw-Hill, New York (1954), Vol. 2, 181-200.

[8] E.M. Ferreira, A.K. Kohara, J. Sesma, New properties of the Lerch's transcendent. J. Number Theory 172 (2017), 21-31; DOI: 10.1016/j.jnt.2016.08.013.

[9] N. Grossman, Polygamma functions of arbitrary order. SIAM J. Math. Anal. 7, No 3 (1976), 366-372.

[10] N. Grossman, Errata: Polygamma functions of arbitrary order. SIAM J. Math. Anal. 8, No 5 (1977), 922.

[11] J. Guillera and J. Sondow, Double integrals and infinite products for some classical constants via analytic continuations of Lerch's transcendent. $R a-$ manujan J. 16 (2008), 247-270; arXiv: math/0506319[math.NT].

[12] H. Hilfer, Threefold Introduction to Fractional Derivatives. In: Anomalous Transport: Foundations and Applications, Wiley-VCH, Weinheim (2008), $17-74$. 
[13] V. Kiryakova, All the special functions are fractional differintegrals of elementary functions. J. Phys. A: Math. Gen. 30 (1997), 5085-5103.

[14] V. Kiryakova, The special functions of fractional calculus as generalized fractional calculus operators of some basic functions. Comput. Math. Appl. 59, No 3 (2010), 1128-1141.

[15] V. Kiryakova, Fractional calculus operators of special functions? The result is well predictable! Chaos Solitons Fractals 102 (2017), 2-15.

[16] J.L. Lavoie, T.J. Osler, R. Tremblay, Fractional derivatives and special functions. SIAM Review 18, No 2 (1976), 240-268.

[17] J.A.T. Machado, V. Kiryakova, The chronicles of fractional calculus. Fract. Calc. Appl. Anal. 20, No 2 (2017), 307-336; DOI: 10.1515/fca-2017-0017.

[18] F.W.J. Olver, D.W. Lozier, R.F. Boisvert, C.W. Clark, NIST Handbook of Mathematical Functions. Cambridge University Press, New York (2010). Available at http://dlmf.nist.gov.

[19] S. Samko, A. Kilbas, O. Marichev, Fractional Integrals and Derivatives. Gordon and Breach, Berlin (1993).

[20] D. Valério, J.J. Trujillo, M. Rivero, J.A.T. Machado, D. Baleanu, Fractional calculus: A survey of useful formulas Eur. Phys. J. Special Topics 222, No 8 (2013), 1827-1846; DOI: 10.1140/epjst/e2013-01967-y. 